\title{
Pseudo-Spline Subdivision Surfaces
}

\author{
Chongyang Deng ${ }^{1}$ and Kai Hormann ${ }^{2}$ \\ ${ }^{1}$ School of Science, Hangzhou Dianzi University, Hangzhou 310018, China \\ ${ }^{2}$ Faculty of Informatics, Università della Svizzera italiana, 6904 Lugano, Switzerland
}

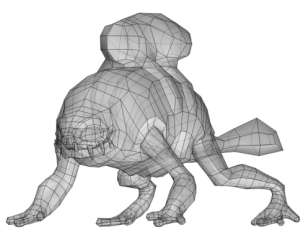

control mesh

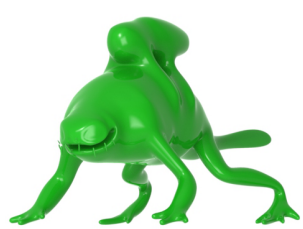

$(n, l)=(4,0), C^{6}$

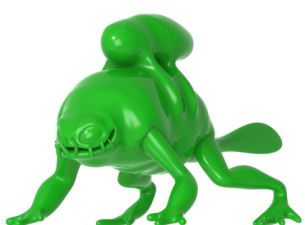

$(n, l)=(4,1), C^{5}$

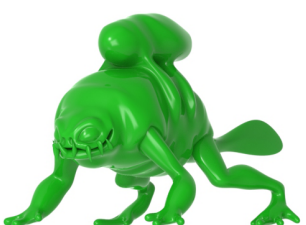

$(n, l)=(4,2), C^{4}$

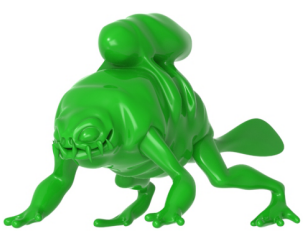

$(n, l)=(4,3), C^{3}$

Figure 1: Pseudo-spline surfaces are a novel family of subdivision schemes for quadrilateral meshes with arbitrary topology. They allow to blend between approximating and interpolatory limit surfaces that are arbitrarily smooth around regular and $C^{1}$ at extraordinary vertices.

\begin{abstract}
Pseudo-splines provide a rich family of subdivision schemes with a wide range of choices that meet various demands for balancing the approximation power, the length of the support, and the regularity of the limit functions. Special cases of pseudo-splines include uniform odd-degree B-splines and the interpolatory $2 n$-point subdivision schemes, and the other pseudo-splines fill the gap between these two families. In this paper we show how the refinement step of a pseudo-spline subdivision scheme can be implemented efficiently using repeated local operations, which require only the data in the direct neighbourhood of each vertex, and how to generalize this concept to quadrilateral meshes with arbitrary topology. The resulting pseudo-spline surfaces can be arbitrarily smooth in regular mesh regions and $C^{1}$ at extraordinary vertices as our numerical analysis reveals.
\end{abstract}

Categories and Subject Descriptors (according to ACM CCS): I.3.5 [Computer Graphics]: Computational Geometry and Object Modeling-Curve, surface, solid, and object representations

\section{Introduction}

Subdivision curves and surfaces are widely used in computer graphics, geometric modelling, and computer animation, because they allow the user to model smooth curves and surfaces by manipulating a small set of control vertices, similar to NURBS modelling. However, unlike NURBS, there is no constraint on the connectivity of the control vertices in the surface setting, which makes subdivision more appropriate for modelling complex surfaces.

Subdivision schemes with higher order continuity usually produce visually smoother surfaces than those with lower order, but they have a larger support and their implementation has to face two difficulties: on the one hand, the computation can be inefficient if the relevant subdivision masks are not properly designed, and on the other hand, complex subdivision masks need to be handled for computing new vertices near extraordinary vertices. In some special cases, these two problems can be solved by using repeated local operations, which require only the data in the direct neighbourhood of a vertex.

The most prominent example is the Lane-Riesenfeld algorithm [LR80], which efficiently refines the control polygon of a uniform B-spline curve of arbitrary degree using only local averaging operations. This idea was later extended to surfaces for refining quadrilateral control meshes of arbitrary topology [Pra98, ZS01, WW01, Sta01]. In regular regions, all these schemes generate tensor-product B-spline 
surfaces of any specified degree [Cas12] and Prautzsch and Chen [PC11] give a rigourous proof of the $C^{1}$-continuity at extraordinary vertices in case of midpoint subdivision for all degrees $d \geq 2$. Similar methods were developed for subdivision of triangular meshes [Sta01], $\sqrt{3}$-subdivision [Kob00, OS03], and nonuniform B-spline surfaces [CADS09]. All these schemes generate limit surfaces that approximate the initial control mesh.

For interpolatory subdivision, Schaefer and Warren [SW03] decompose the 4-point scheme [DGL87] into several simple passes, which are similar to those of the LaneRiesenfeld algorithm. Deng and Ma [DM13] extend this idea and show how to factor the general interpolatory $2 n$-point subdivision scheme [DD89] into repeated local operations and generalize their approach to quadrilateral meshes. However, in contrast to approximating schemes, where the direct neighbour information is used in each local step, Deng and Ma's method uses the information of two neighbouring edges for updating new vertices. Moreover, they use two rounds, one for new edge vertices and one for new face vertices, both based on the rules from the curve case.

Approximating schemes usually lead to visually smoother limit surfaces than interpolating schemes, but they come with the disadvantage of being harder to model, as the limit surface can be relatively far from the control vertices. In the context of solving the "popping effect" when switching between meshes at different levels of resolution, Maillot and Stam [MS01] propose a unified subdivision scheme which blends approximating spline-based schemes with interpolatory subdivision schemes. Rossignac and Schaefer [RS08] then explore the continuity of such splines and extend it to open curves and smooth surfaces for quadrilateral meshes with arbitrary connectivity. The idea is also used to design new interpolatory and blending subdivision schemes [LM07, LLYL08]. However, all these approaches do not consider interpolatory or blending schemes with higher order.

Pseudo-splines provide an alternative to naturally blend between interpolatory and approximating schemes. More precisely, we consider pseudo-splines of type II, which were introduced by Dong and Shen [DS07] to construct symmetric or antisymmetric tight framelets with desired approximation orders. Depending on the choice of the two parameters that these pseudo-splines are equipped with, they include uniform odd-degree B-splines, the interpolatory $2 n$ point subdivision schemes, and a wide range of schemes in between. Hence, they provide a rich family of schemes that meet various demands for balancing the approximation power, the length of the support, and the regularity of the limit curves [DHSS08].

In this paper we first present a framework for implementing pseudo-spline curves efficiently with repeated local operations (see Section 2) and then generalize this concept to quadrilateral meshes with arbitrary topology (see Section 3), similar to Stam's method for generalizing B-splines [Sta01].
In a nutshell, each subdivision step first refines the control mesh with a simple refinement subdivision step and then updates the vertices of the refined mesh with a number of local operations that depend only on the direct neighbourhood of each vertex. This strategy is more general than the one described by Deng and Ma [DM13] and it is also simpler, because after the initial refinement stage, all new vertices are treated in the same way, so that there is no need to distinguish between different vertex types. The resulting pseudo-spline surfaces are tensor products of univariate pseudo-splines around regular vertices, thus inheriting their order of continuity, and around extraordinary vertices the limit surface turns out to be $C^{1}$ (see Section 4). The pseudospline parameters then provide the user with intuitive control for selecting a limit surface that is visually smooth and still close to the initial control mesh (see Section 5).

The main contributions of this paper are:

- decomposing the pseudo-spline refinement rule into repeated local operations;

- extending this idea to quadrilateral meshes;

- developing special local rules for extraordinary vertices which guarantee the limit surfaces to be $C^{1}$-continuous.

\section{Pseudo-spline curves}

Starting from some initial control polygon $\boldsymbol{P}^{0}$ with vertices $\boldsymbol{P}_{i}^{0}, i \in \mathbb{Z}$, a subdivision scheme generates the refined polygons $\boldsymbol{P}^{k}, k \in \mathbb{N}$ recursively according to the refinement equation

$$
\boldsymbol{P}_{i}^{k+1}=\sum_{j \in \mathbb{Z}} a_{i-2 j} \boldsymbol{P}_{j}^{k}, \quad i \in \mathbb{Z},
$$

where $\boldsymbol{a}=\left(a_{i}\right)_{i \in \mathbb{Z}}$ is the subdivision mask. The z-transform of the mask $\boldsymbol{a}$,

$$
a(z)=\sum_{i \in \mathbb{Z}} a_{i} z^{i},
$$

is usually called the symbol of the scheme.

The pseudo-spline schemes are defined by the symbols [DS07, DHSS08]

$$
a_{n}^{l}(z)=2 \sigma(z)^{n} \sum_{j=0}^{l}\left(\begin{array}{c}
n+j-1 \\
j
\end{array}\right) \delta(z)^{j},
$$

where

$$
\sigma(z)=\frac{(1+z)^{2}}{4 z} \quad \text { and } \quad \delta(z)=-\frac{(1-z)^{2}}{4 z} .
$$

Note that $a_{n}^{0}(z)=2 \sigma(z)^{n}$ is the symbol of the $(2 n-1)$ degree uniform B-spline scheme with $C^{2 n-2}$-continuous limit curves [LR80] and that $a_{n}^{n-1}(z)$ is the symbol of the interpolatory $2 n$-point subdivision scheme [DD89] with $C^{r_{n}}$ continuous limit curves, where $r_{n} \approx 0.415 n$ [Dau92, Eir92]. For fixed $n$, the regularity of the limit curves decreases as $l$ increases, and for fixed $l$ the regularity increases with $n$ [DS07, FM13]. 


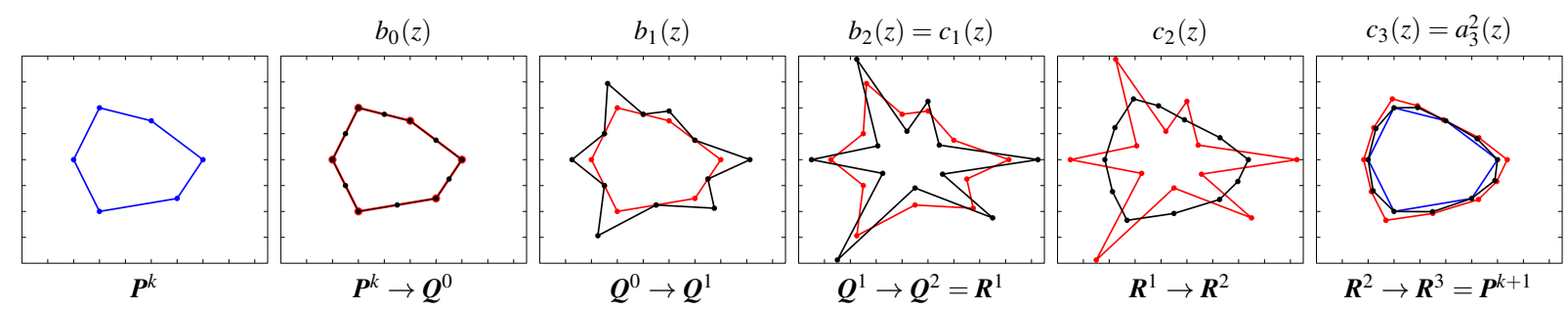

Figure 2: Splitting the subdivision step of the pseudo-spline with symbol $a_{3}^{2}(z)$ to refine $\boldsymbol{P}^{k}$ to $\boldsymbol{P}^{k+1}$ into 5 repeated local operations. In each step, the polygon before the operation is shown in red and the polygon after the operation in black.

Since multiplying the symbol with the smoothing factor $\sigma(z)$ corresponds to updating each vertex with the weighted average of itself and its two neighbours [WW01], the crucial point of implementing the subdivision step of the pseudospline with symbol $a_{n}^{l}(z)$ with repeated local operations is the decomposition of

$$
b_{l}(z)=2 \sigma(z) \sum_{j=0}^{l}\left(\begin{array}{c}
n+j-1 \\
j
\end{array}\right) \delta(z)^{j}
$$

into a sequence of local operations.

Theorem 1 The symbol $b_{m}(z)$ satisfies the recursive formula

$$
b_{m+1}(z)=b_{m}(z)+\gamma_{m} \delta(z) d_{m}(z)
$$

with $d_{m}(z)=b_{m}(z)-b_{m-1}(z)$ and $\gamma_{m}=(n+m) /(m+1)$.

Proof By the definition of $b_{m}(z)$ in (2),

$$
d_{m}(z)=b_{m}(z)-b_{m-1}(z)=2 \sigma(z)\left(\begin{array}{c}
n+m-1 \\
m
\end{array}\right) \delta(z)^{m},
$$

and (3) then follows because

$$
\begin{aligned}
b_{m+1}(z)-b_{m}(z) & =2 \sigma(z)\left(\begin{array}{c}
n+m \\
m+1
\end{array}\right) \delta(z)^{m+1} \\
& =\gamma_{m} \delta(z) d_{m}(z) .
\end{aligned}
$$

Following Theorem 1 and letting

$$
c_{m}(z)=\sigma(z)^{m-1} b_{l}(z),
$$

the symbol $a_{n}^{l}(z)$ is given by the sequence

$$
\begin{aligned}
& 2 \sigma(z)=b_{0}(z) \rightarrow b_{1}(z) \rightarrow \cdots \rightarrow b_{l}(z) \\
& a_{n}^{l}(z)=c_{n}(z) \leftarrow \cdots \leftarrow c_{2}(z) \leftarrow c_{1}(z)
\end{aligned}
$$

where the arrows in the top row correspond to the recursive formula (3) and the arrows in the bottom row correspond to a multiplication with $\sigma(z)$.

This sequence leads to the following computations for subdividing the polygon $\boldsymbol{P}^{k}$. We first refine $\boldsymbol{P}^{k}$ by inserting all edge midpoints, giving the intermediate polygon $Q^{0}$ with vertices

$$
\boldsymbol{Q}_{2 j}^{0}=\boldsymbol{P}_{j}^{k}, \quad \boldsymbol{Q}_{2 j+1}^{0}=\left(\boldsymbol{P}_{j}^{k}+\boldsymbol{P}_{j+1}^{k}\right) / 2, \quad j \in \mathbb{Z} .
$$

This refinement corresponds to the initial symbol $b_{0}(z)=$ $2 \sigma(z)$ in (5). We now proceed to update the vertices of

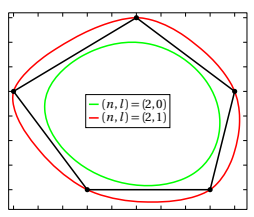

$n=2$

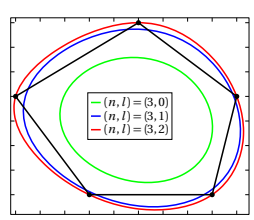

$n=3$

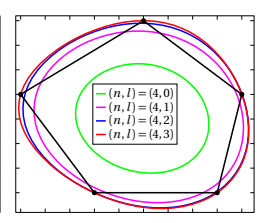

$n=4$
Figure 3: Examples of pseudo-spline curves defined by the black initial control polygon.

$Q^{0}$ by iteratively applying the $l$ local operations as derived from (3),

$$
\begin{aligned}
\boldsymbol{D}_{j}^{m} & =\boldsymbol{Q}_{j}^{m}-\boldsymbol{Q}_{j}^{m-1}, \\
\boldsymbol{Q}_{j}^{m+1} & =\boldsymbol{Q}_{j}^{m}+\gamma_{m}\left(-\boldsymbol{D}_{j-1}^{m}+2 \boldsymbol{D}_{j}^{m}-\boldsymbol{D}_{j+1}^{m}\right) / 4,
\end{aligned}
$$

for $j \in \mathbb{Z}$ and $m=0, \ldots, l-1$, with $\boldsymbol{Q}_{j}^{-1}=\mathbf{0}$. After these $l$ steps we obtain the intermediate polygon $\boldsymbol{Q}^{l}$ which corresponds to a subdivision step with the symbol $b_{l}(z)=c_{1}(z)$.

It remains to set $\boldsymbol{R}_{j}^{1}=\boldsymbol{Q}_{j}^{l}$ and to carry out the $n-1$ local operations which relate to the bottom row of (5) by iteratively computing

$$
\boldsymbol{R}_{j}^{m+1}=\left(\boldsymbol{R}_{j-1}^{m}+2 \boldsymbol{R}_{j}^{m}+\boldsymbol{R}_{j+1}^{m}\right) / 4
$$

for $j \in \mathbb{Z}$ and $m=1, \ldots, n-1$, to finally get the vertices $\boldsymbol{P}_{j}^{k+1}=\boldsymbol{R}_{j}^{n}$ of the refined polygon $\boldsymbol{P}^{k+1}$.

Figure 2 illustrates this procedure for the interpolatory 6-point subdivision scheme, which is the pseudo-spline scheme with symbol $a_{3}^{2}(z)$. Figure 3 further shows some examples of limit curves for the pseudo-spline schemes. Remember that the choice $l=0$ gives uniform B-splines of degree $2 n-1$ (green curves) and that $l=n-1$ gives the limit curves of the interpolatory $2 n$-point scheme (red curves), and note how varying $l$ blends between these two limit curves. It is even possible to consider non-integer values of $l$ by adapting the ideas in [RS08] for continuously blending between the subdivision rules of integer-valued pseudo-spline schemes, but investigating this approach, which would also work in the surface setting, is beyond the scope of this paper.

Since each local operation enlarges the support by 1 both to the left and to the right, it is not hard to see that the support of the basic limit function of the pseudo-spline with symbol 
C. Deng \& K. Hormann / Pseudo-Spline Subdivision Surfaces

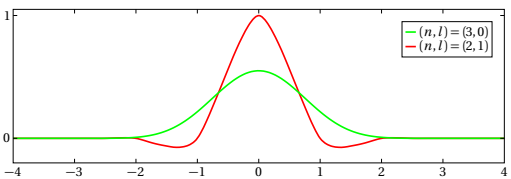

support $[-3,3]$

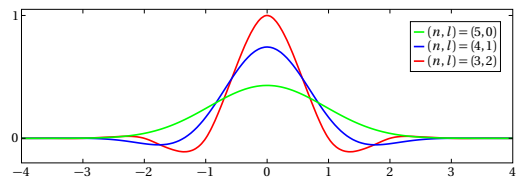

support $[-5,5]$

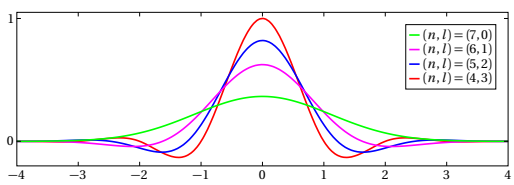

support $[-7,7]$

Figure 4: Examples of basic limit functions for several pseudo-splines, grouped by the size of their support.

$a_{n}^{l}(z)$ is $[-n-l, n+l]$, but Figure 4 shows that the practical support is considerably smaller.

\section{Pseudo-spline surfaces}

The same idea can also be applied to efficiently compute pseudo-spline surfaces from quadrilateral control meshes. Let us first consider the tensor product setting, where all vertices $\boldsymbol{P}_{i, j}^{0}, i, j \in \mathbb{Z}$ of the initial control mesh $\boldsymbol{M}^{0}$ are regular with valency 4 and the vertices of the refined meshes $\boldsymbol{M}^{k}$, $k \in \mathbb{N}$ are generated recursively by the refinement equation

$$
\boldsymbol{P}_{i, j}^{k+1}=\sum_{m, n \in \mathbb{Z}} a_{i-2 m, j-2 n} \boldsymbol{P}_{i, j}^{k}, \quad i, j \in \mathbb{Z},
$$

where $\boldsymbol{a}=\left(a_{i, j}\right)_{i, j} \in \mathbb{Z}^{2}$ is the bivariate subdivision mask and its $z$-transform

$$
\boldsymbol{a}(x, y)=\sum_{i, j \in \mathbb{Z}} a_{i, j} x^{i} y^{j}
$$

is the bivariate symbol of the scheme. Defining the bivariate symbols

$$
\begin{aligned}
& \boldsymbol{\sigma}(x, y)=\boldsymbol{\sigma}(x) \boldsymbol{\sigma}(y), \\
& \boldsymbol{\delta}(x, y)=\boldsymbol{\delta}(x) \boldsymbol{\delta}(y), \\
& \boldsymbol{\chi}(x, y)=\boldsymbol{\delta}(x)+\boldsymbol{\delta}(y)
\end{aligned}
$$

and

$$
\begin{aligned}
& \boldsymbol{b}_{m}(x, y)=b_{m}(x) b_{m}(y), \\
& \boldsymbol{d}_{m}(x, y)=d_{m}(x) d_{m}(y), \\
& \boldsymbol{e}_{m}(x, y)=\boldsymbol{b}_{m}(x, y)-\boldsymbol{b}_{m-1}(x, y),
\end{aligned}
$$

the symbol of the tensor product pseudo-spline scheme can be written as

$$
\boldsymbol{a}_{n}^{l}(x, y)=a_{n}^{l}(x) a_{n}^{l}(y)=\boldsymbol{\sigma}(x, y)^{n-1} \boldsymbol{b}_{l}(x, y)
$$

and, like in the curve case, the crucial step is to decompose the symbol $\boldsymbol{b}_{l}(x, y)$ into a sequence of local operations.

Theorem 2 The symbol $\boldsymbol{b}_{m}(x, y)$ satisfies the recursive formula

$$
\begin{aligned}
\boldsymbol{b}_{m+1}(x, y)= & \boldsymbol{b}_{m}(x, y)+\boldsymbol{d}_{m+1}(x, y) \\
& -\left(\gamma_{m} / \gamma_{m-1}\right) \boldsymbol{d}_{m}(x, y) \\
& +\gamma_{m} \boldsymbol{\chi}(x, y) \boldsymbol{e}_{m}(x, y) \\
& -\gamma_{m} \gamma_{m-1} \boldsymbol{\delta}(x, y) \boldsymbol{e}_{m-1}(x, y) .
\end{aligned}
$$

Proof By Theorem 1 and noting that (4) implies

$$
\boldsymbol{d}_{m+1}(x, y)=\gamma_{m}{ }^{2} \boldsymbol{\delta}(x, y) \boldsymbol{d}_{m}(x, y),
$$

we have

$$
\begin{aligned}
\boldsymbol{b}_{m+1} & (x, y) \\
= & \left(b_{m}(x)+\gamma_{m} \delta(x)\left[b_{m}(x)-b_{m-1}(x)\right]\right) \\
& \times\left(b_{m}(y)+\gamma_{m} \delta(y)\left[b_{m}(y)-b_{m-1}(y)\right]\right) \\
= & \boldsymbol{b}_{m}(x, y)+\boldsymbol{d}_{m+1}(x, y)+\gamma_{m} \boldsymbol{\chi}(x, y) \boldsymbol{b}_{m}(x, y) \\
& -\gamma_{m}\left[b_{m}(x) \boldsymbol{\delta}(y) b_{m-1}(y)+b_{m}(y) \boldsymbol{\delta}(x) b_{m-1}(x)\right] .
\end{aligned}
$$

Equation (6) then follows because

$$
\begin{aligned}
b_{m}(x) & \delta(y) b_{m-1}(y)+b_{m}(y) \delta(x) b_{m-1}(x) \\
= & {\left[b_{m-1}(x)+\gamma_{m-1} \delta(x) d_{m-1}(x)\right] \delta(y) b_{m-1}(y) } \\
& +\left[b_{m-1}(y)+\gamma_{m-1} \delta(y) d_{m-1}(y)\right] \delta(x) b_{m-1}(x) \\
= & \boldsymbol{\chi}(x, y) \boldsymbol{b}_{m-1}(x, y) \\
& +\gamma_{m-1} \boldsymbol{\delta}(x, y)\left[d_{m-1}(x) b_{m-1}(y)+d_{m-1}(y) b_{m-1}(x)\right]
\end{aligned}
$$

and, using again (7),

$$
\begin{array}{r}
d_{m-1}(x) b_{m-1}(y)+d_{m-1}(y) b_{m-1}(x) \\
=\boldsymbol{b}_{m-1}(x, y)+\boldsymbol{d}_{m-1}(x, y)-\boldsymbol{b}_{m-2}(x, y) \\
=\boldsymbol{e}_{m-1}(x, y)+\boldsymbol{d}_{m}(x, y) /\left[\gamma_{m-1}{ }^{2} \boldsymbol{\delta}(x, y)\right] .
\end{array}
$$

Therefore, letting

$$
\boldsymbol{c}_{m}(x, y)=c_{m}(x) c_{m}(y)=\boldsymbol{\sigma}(x, y)^{m-1} \boldsymbol{b}_{l}(x, y),
$$

the symbol $\boldsymbol{a}_{n}^{l}(x, y)$ is given by the sequence

$$
\begin{aligned}
& 4 \boldsymbol{\sigma}(x, y)=\boldsymbol{b}_{0}(x, y) \rightarrow \boldsymbol{b}_{1}(x, y) \rightarrow \cdots \rightarrow \boldsymbol{b}_{l}(x, y) \\
& \boldsymbol{a}_{n}^{l}(x, y)=\boldsymbol{c}_{n}(x, y) \leftarrow \cdots \leftarrow \boldsymbol{c}_{2}(x, y) \leftarrow \boldsymbol{c}_{1}(x, y)
\end{aligned}
$$

similar to how it is done in the univariate setting in (5).

In the tensor product setting,

$$
\begin{aligned}
& \boldsymbol{\sigma}(x, y)=\frac{1}{4}+\frac{1}{8}\left(x+\frac{1}{x}+y+\frac{1}{y}\right)+\frac{1}{16}\left(x y+\frac{1}{x y}+\frac{x}{y}+\frac{y}{x}\right), \\
& \boldsymbol{\delta}(x, y)=\frac{1}{4}-\frac{1}{8}\left(x+\frac{1}{x}+y+\frac{1}{y}\right)+\frac{1}{16}\left(x y+\frac{1}{x y}+\frac{x}{y}+\frac{y}{x}\right), \\
& \boldsymbol{\chi}(x, y)=1-\frac{1}{4}\left(x+\frac{1}{x}+y+\frac{1}{y}\right),
\end{aligned}
$$

which corresponds to the masks shown in Figure 6.

For an extraordinary vertex with valency $N$, we adopt the weights used in the repeated averaging algorithm for the surface generalization of odd-degree B-spline subdivision [ZS01] and generalize the mask $\boldsymbol{\sigma}(x, y)$ as shown in Figure 7. Following the pattern of the regular masks, we set the weights of the generalized mask $\boldsymbol{\delta}(x, y)$ to the same values, 


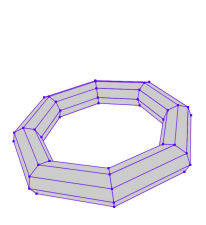

$\boldsymbol{P}^{k}$

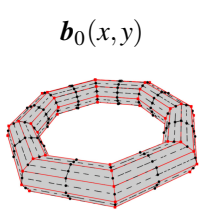

$\boldsymbol{P}^{k} \rightarrow \boldsymbol{Q}^{0}$

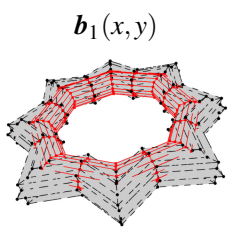

$Q^{0} \rightarrow Q^{1}$

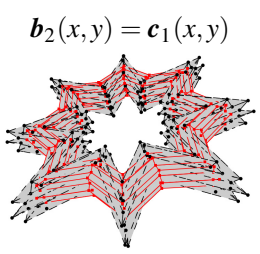

$Q^{1} \rightarrow Q^{2}=R^{1}$

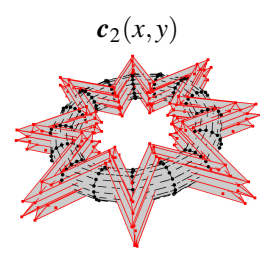

$\boldsymbol{R}^{1} \rightarrow \boldsymbol{R}^{2}$ $\boldsymbol{c}_{3}(x, y)=\boldsymbol{a}_{3}^{2}(x, y)$

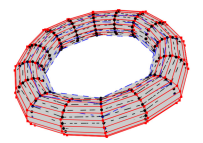

$\boldsymbol{R}^{2} \rightarrow \boldsymbol{R}^{3}=\boldsymbol{P}^{k+1}$

Figure 5: Splitting the subdivision step of the pseudo-spline with symbol $\boldsymbol{a}_{3}^{2}(x, y)$ to refine $\boldsymbol{P}^{k}$ to $\boldsymbol{P}^{k+1}$ into 5 repeated local operations. In each step, the mesh before the operation is shown in red and the mesh after the operation in black.

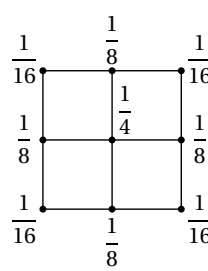

$\boldsymbol{\sigma}(x, y)$

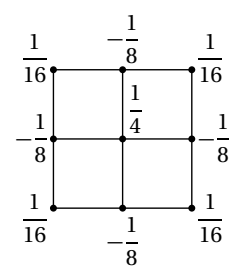

$\boldsymbol{\delta}(x, y)$

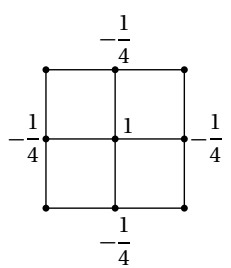

$\chi(x, y)$
Figure 6: Masks for local operations at regular vertices.

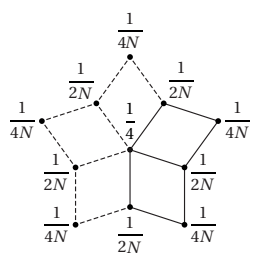

$\boldsymbol{\sigma}(x, y)$

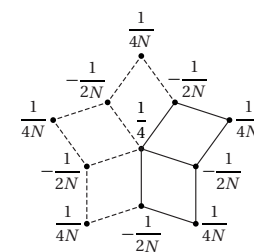

$\boldsymbol{\delta}(x, y)$

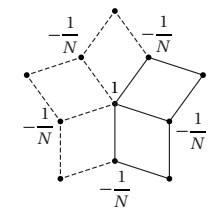

$\boldsymbol{\chi}(x, y)$
Figure 7: Masks for local operations at extraordinary vertices.

except that we negate the weights of the direct neighbours, and an intuitive generalization of the mask for $\boldsymbol{\chi}(x, y)$ is the one in Figure 7. Note that these generalized masks are identical to the masks in Figure 6 for $N=4$ and satisfy the identity

$$
\boldsymbol{\delta}(x, y)=\boldsymbol{\sigma}(x, y)+\boldsymbol{\chi}(x, y)-1,
$$

just as in the regular setting.

According to Theorem 2 and the sequence diagram in (8), we perform the following computations for refining the mesh $\boldsymbol{M}^{k}$ with vertices $\boldsymbol{P}_{j}^{k}$. Analogously to the curve setting, we first refine $\boldsymbol{M}^{k}$ by inserting all edge midpoints and the barycentres of all faces, giving the intermediate mesh $N^{0}$ with vertices

$$
\boldsymbol{Q}_{j}^{0}=\boldsymbol{P}_{j}^{k}
$$

copied from $\boldsymbol{M}^{k}$, new edge vertices

$$
\boldsymbol{Q}_{e}^{0}=\left(\boldsymbol{P}_{e_{1}}^{k}+\boldsymbol{P}_{e_{2}}^{k}\right) / 2,
$$

one for each edge $e=\left[\boldsymbol{P}_{e_{1}}^{k}, \boldsymbol{P}_{e_{2}}^{k}\right]$ in $\boldsymbol{M}^{k}$, and new face vertices

$$
\boldsymbol{Q}_{f}^{0}=\left(\boldsymbol{P}_{f_{1}}^{k}+\boldsymbol{P}_{f_{2}}^{k}+\boldsymbol{P}_{f_{3}}^{k}+\boldsymbol{P}_{f_{4}}^{k}\right) / 4,
$$

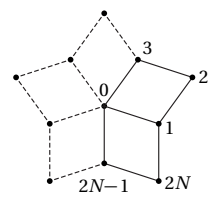

Figure 8: Local indices of a vertex and its neighbours.

one for each quadrilateral $f=\left[\boldsymbol{P}_{f_{1}}^{k}, \boldsymbol{P}_{f_{2}}^{k}, \boldsymbol{P}_{f_{3}}^{k}, \boldsymbol{P}_{f_{4}}^{k}\right]$ in $\boldsymbol{M}^{k}$. The vertices of $\boldsymbol{N}^{0}$ are connected by splitting the quadrilaterals in $\boldsymbol{M}^{k}$ with the classical 1-to-4 rule, so that $\boldsymbol{N}^{0}$ is again a quadrilateral mesh. This refinement corresponds to the initial symbol $\boldsymbol{b}_{0}(x, y)=4 \boldsymbol{\sigma}(x, y)$ in (8), and we now proceed to update the vertices of $\boldsymbol{N}^{0}$ by iteratively applying the $l$ local operations as derived from (6) to all vertices.

Without loss of generality let $\boldsymbol{Q}_{0}^{0}$ be a vertex in $\boldsymbol{N}^{0}$ with valency $N$ and neighbours $\boldsymbol{Q}_{1}^{0}, \ldots, \boldsymbol{Q}_{2 N}^{0}$ as shown in Figure 8 . Equations (7) and (6) then translate to computing

$$
\begin{aligned}
\boldsymbol{D}_{0}^{m+1}= & \frac{\gamma_{m}^{2}}{N} \sum_{i=1}^{N}\left(\boldsymbol{D}_{0}^{m}-2 \boldsymbol{D}_{2 i-1}^{m}+\boldsymbol{D}_{2 i}^{m}\right) / 4, \\
\boldsymbol{E}_{i}^{m}= & \boldsymbol{Q}_{i}^{m}-\boldsymbol{Q}_{i}^{m-1}, \quad i=0, \ldots, 2 N \\
\boldsymbol{Q}_{0}^{m+1}= & \boldsymbol{Q}_{0}^{m}+\boldsymbol{D}_{0}^{m+1}-\left(\gamma_{m} / \gamma_{m-1}\right) \boldsymbol{D}_{0}^{m} \\
& +\frac{\gamma_{m}}{N} \sum_{i=1}^{N}\left(\boldsymbol{E}_{0}^{m}-\boldsymbol{E}_{2 i-1}^{m}\right) \\
& -\frac{\gamma_{m} \gamma_{m-1}}{N} \sum_{i=1}^{N}\left(\boldsymbol{E}_{0}^{m-1}-2 \boldsymbol{E}_{2 i-1}^{m-1}+\boldsymbol{E}_{2 i}^{m-1}\right) / 4,
\end{aligned}
$$

for $m=0, \ldots, l-1$, with initial values $\boldsymbol{D}_{j}^{0}=\boldsymbol{Q}_{j}^{0}, \boldsymbol{Q}_{j}^{-1}=\mathbf{0}$ and the convention that $\gamma_{0} / \gamma_{-1}=0$ and $\gamma_{-1}=0$. After these $l$ steps we obtain the intermediate mesh $N^{l}$ which corresponds to a subdivision step with the symbol $\boldsymbol{b}_{l}(x, y)=$ $\boldsymbol{c}_{1}(x, y)$.

It remains to to set $\boldsymbol{R}_{j}^{1}=\boldsymbol{Q}_{j}^{l}$ and to carry out the $n-1$ local operations which relate to the bottom row of (8) by iteratively computing

$$
\boldsymbol{R}_{0}^{m+1}=\frac{1}{N} \sum_{i=1}^{N}\left(\boldsymbol{R}_{0}^{m}+2 \boldsymbol{R}_{2 i-1}^{m}+\boldsymbol{R}_{2 i}^{m}\right) / 4
$$


C. Deng \& K. Hormann / Pseudo-Spline Subdivision Surfaces
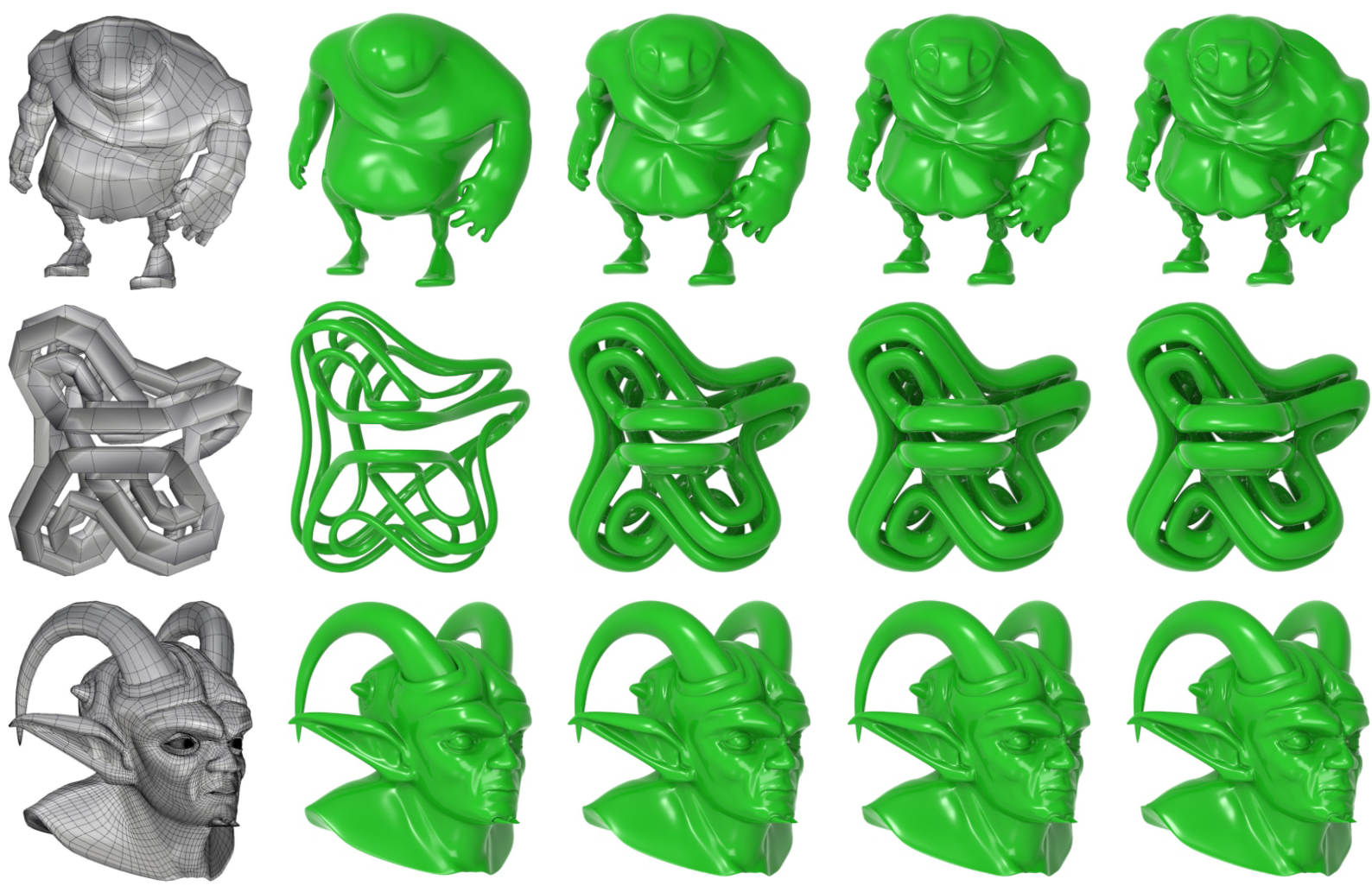

control mesh

$(n, l)=(4,0), C^{6}$

$(n, l)=(4,1), C^{5}$

$(n, l)=(4,2), C^{4}$

$(n, l)=(4,3), C^{3}$

Figure 9: Pseudo-spline surfaces with fixed $n$ and varying $l$.
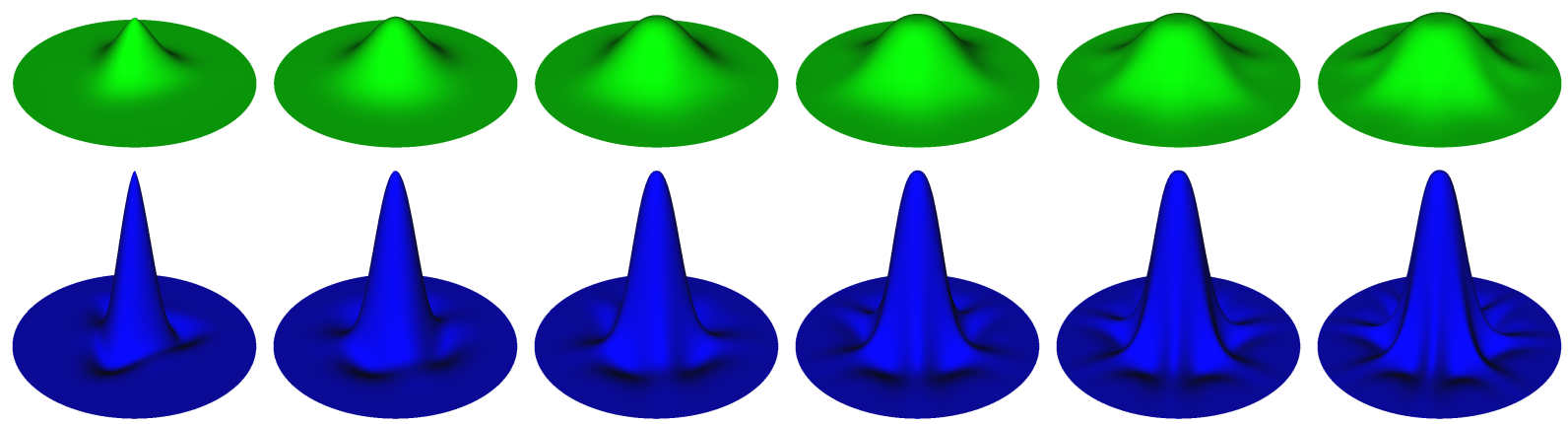

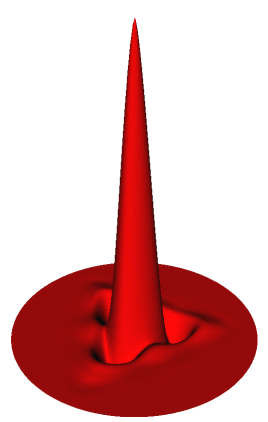

$N=3$

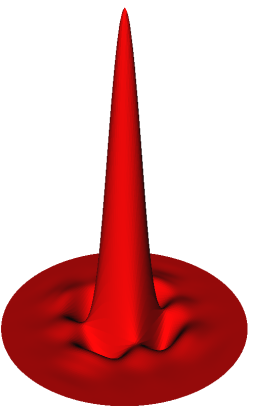

$N=4$

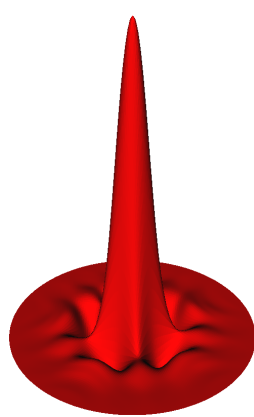

$N=5$

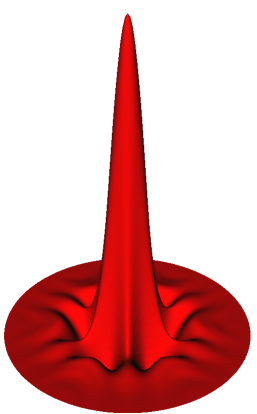

$N=6$

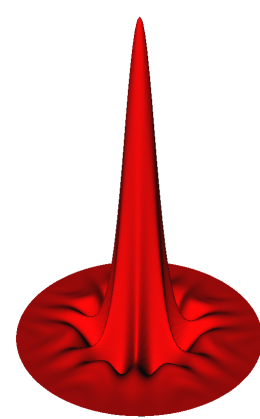

$N=7$

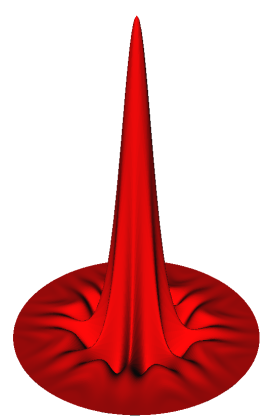

$N=8$

Figure 10: Examples of basic limit functions for different valencies $N$ and pseudo-spline surfaces with $(n, l)=(5,0)($ top $)$, $(n, l)=(4,1)$ (middle), and $(n, l)=(3,2)$ (bottom). All functions have the same support size, namely the 5-ring neighbourhood. 


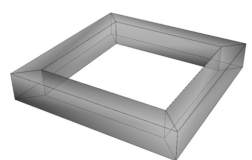

control mesh

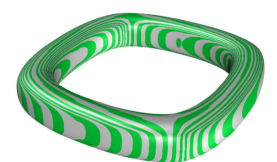

$(n, l)=(2,1), C^{1}$

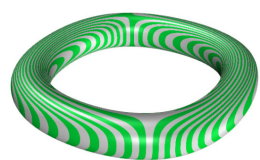

$(n, l)=(3,2), C^{2}$

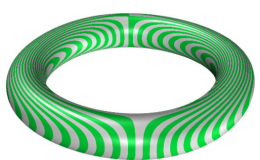

$(n, l)=(4,3), C^{3}$

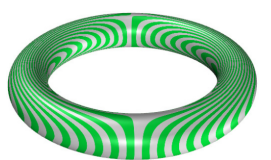

$(n, l)=(5,4), C^{4}$

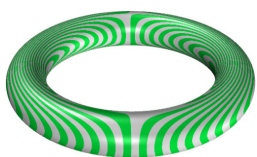

$(n, l)=(6,5), C^{4}$

Figure 11: Interpolatory pseudo-spline surfaces with increasing $n$ and $l=n-1$ for a torus control mesh.
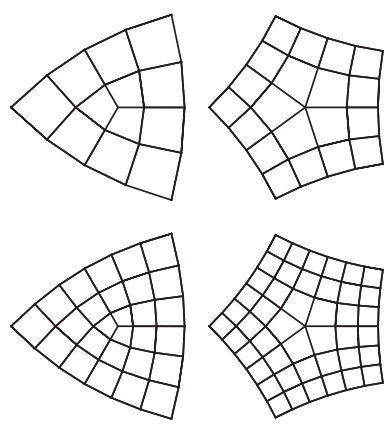

$N=3$
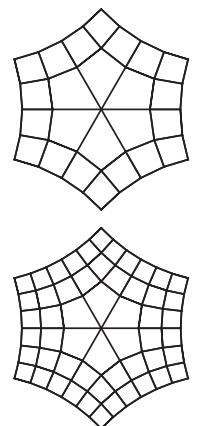

$N=6$
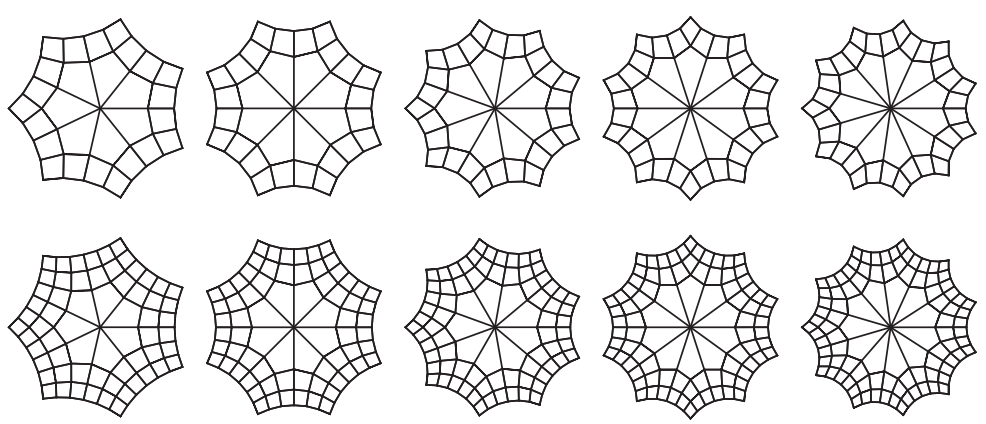

$N=7$

$N=8$

$N=9$

$N=10$

$N=11$

Figure 12: Characteristic maps of the pseudo-splines with $(n, l)=(2,1)$ (top) and $(n, l)=(3,1)$ (bottom) for extraordinary vertices.

for $m=1, \ldots, n-1$, to finally get the vertices $\boldsymbol{P}_{j}^{k+1}=\boldsymbol{Q}_{j}^{n}$ of the refined mesh $\boldsymbol{M}^{k+1}$.

Figure 5 illustrates this procedure for the pseudo-spline subdivision scheme with symbol $\boldsymbol{a}_{3}^{2}(x, y)$, which generalizes the 6-point interpolatory subdivision scheme. Figures 1 and 9 further show some examples of limit surfaces for the pseudo-spline surface schemes.

\subsection{Properties}

Since each local operation enlarges the support exactly by one 1-ring neighbourhood, it follows that the support of the basic limit function of the pseudo-spline surface scheme with parameters $n$ and $l$ extends to the $(n+l)$-ring neighbourhood. Some examples are shown in Figure 10.

Pseudo-spline surfaces generalize several known subdivision schemes. For example, choosing $l=0$ reproduces the approximating B-spline schemes by Zorin and Schröder [ZS01] and the interpolatory scheme by $\mathrm{Li}$ and Ma [LM07] is reproduced for $n=2$ and $l=1$. More generally, the pseudo-spline schemes with $n \geq 2$ and $l=n-1$, which are the surface analogues of the interpolatory $2 n$-point schemes, are identical to the alternative schemes by Deng and Ma [DM13] for regular vertices. Consequently, pseudospline surfaces can generate exact tori in the limit as $n \rightarrow \infty$ as shown in [DM13] and illustrated in Figure 11. Around irregular vertices, the subdivision rules are different and while the limit surfaces of [DM13] interpolate all initial vertices, pseudo-spline surfaces interpolate only regular initial vertices with regular neighbours.
The Catmull-Clark scheme [CC78] can also be reproduced by choosing $n=2$ and $l=0$ and by slightly modifying the generalized mask $\boldsymbol{\sigma}(x, y)$ in Figure 7, that is, by replacing the weights $\frac{1}{2 N}$ of the direct neighbours with $\frac{2}{N^{2}}$ and the weights $\frac{1}{4 N}$ of the opposite neighbours with $\frac{1}{N^{2}}$. However, we found that our choice of $\boldsymbol{\sigma}(x, y)$ gives better curvature behaviour of the limit surfaces for general $n$ and $l$.

We actually tried different ways of generalizing the masks in Figure 6 for extraordinary vertices, using the obvious symmetries of the weights, the summation of weights to one for $\boldsymbol{\sigma}$ and $\boldsymbol{\chi}$ and to zero for $\boldsymbol{\delta}$, and identity (9) as constraints, but found that the choice of weights given in Figure 7 performed best.

\section{Smoothness analysis}

The smoothness of pseudo-spline surfaces at regular vertices is the same as that of the corresponding univariate pseudospline and increases for increasing $n$ and decreasing $l$. At extraordinary vertices, we verified numerically that the surfaces are $C^{1}$-continuous for $2 \leq n \leq 5,1 \leq l \leq n-1$ and valency $3 \leq N \leq 50$.

More precisely, we follow [PR08] and first used Matlab to verify that the leading eigenvalues of the subdivision matrix satisfy

$$
1=\lambda_{0}>\lambda_{1}=\lambda_{2}>\left|\lambda_{3}\right| \text {. }
$$

Secondly, we checked that the characteristic maps are regular, using Theorem 5.25 of [PR08] and a numerical checking 

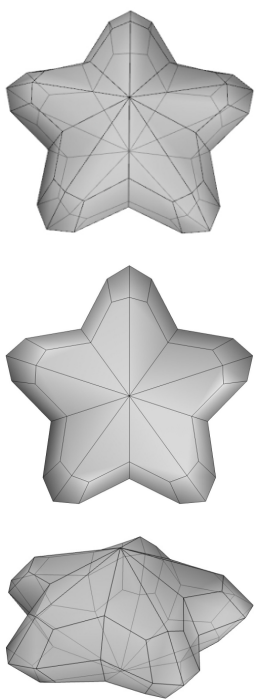

control mesh
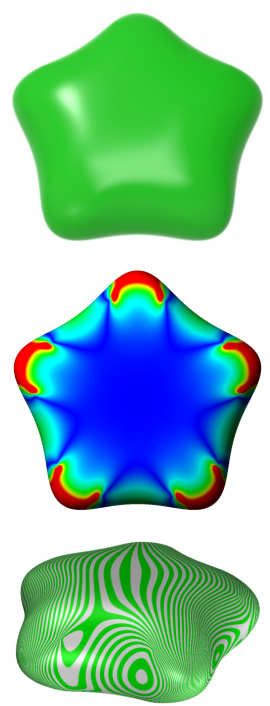

$(n, l)=(4,0), C^{6}$
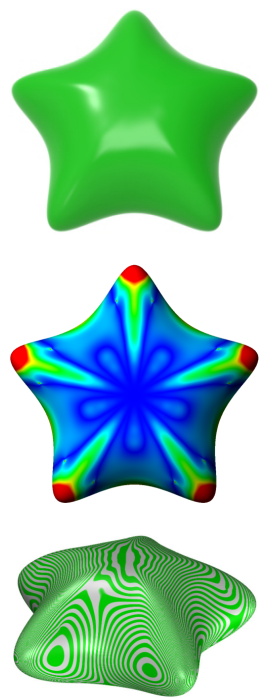

$(n, l)=(4,1), C^{5}$
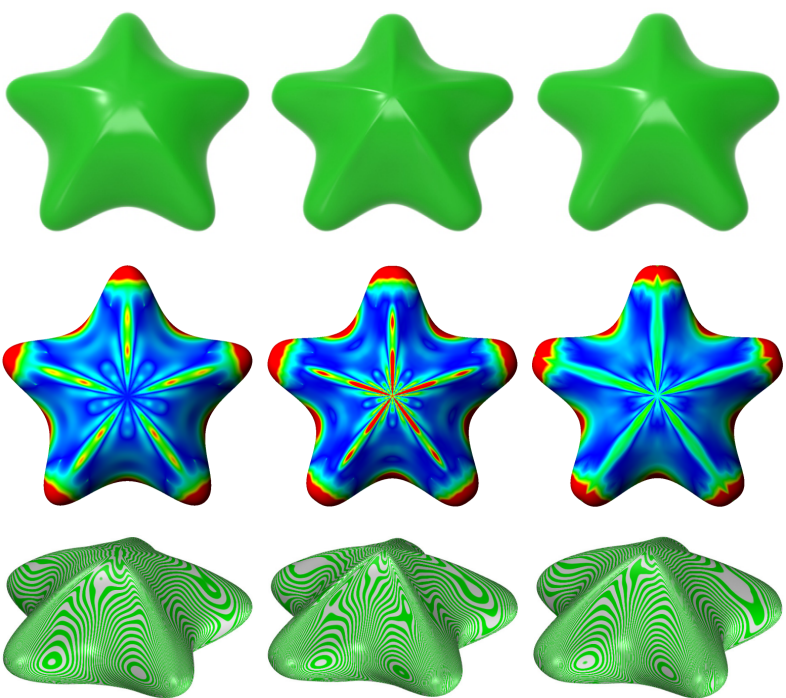

$(n, l)=(4,2), C^{4}$

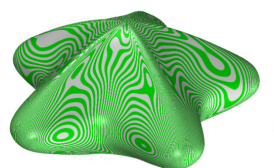

$(n, l)=(4,3), C^{3}$

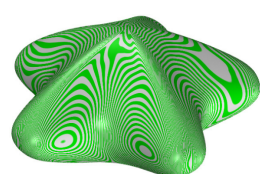

8-point, $C^{3}$

Figure 13: Pseudo-spline surfaces with fixed $n$ and varying $l$ and the 8-point surface of Deng and Ma [DM13] for a star-shaped control mesh.
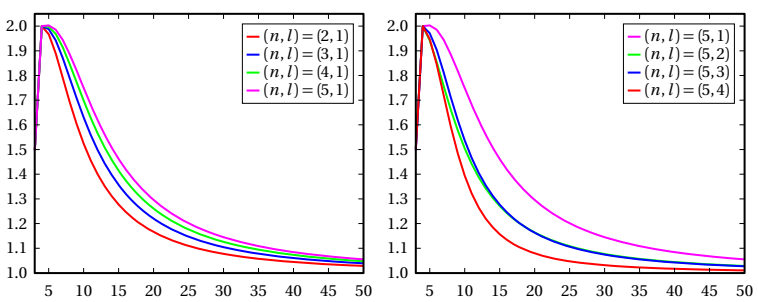

Figure 14: Plot of $\log \lambda_{2} / \log \lambda_{1}$ over $N$ for various $n$ and $l$.

method similar to that of Augsdörfer et al. [ACDS09]. Figure 12 shows some typical examples of these characteristic maps. Thirdly, we checked that the Fourier indices [PR04] of the subdominant eigenvalue $\lambda_{1}$ are $\{1, N-1\}$, which then guarantees the injectivity of the characteristic maps. Overall, this is sufficient to conclude $C^{1}$-continuity of the limit surface.

At extraordinary vertices, our schemes do not satisfy the necessary condition $\left(\lambda_{1}\right)^{2}=\lambda_{2}$ for bounded curvature and curvature continuity and so the limit surfaces are not $C^{2}$. However, the smoothness improves for increasing $n$ and $l$, because $\lambda_{2}$ approaches $\left(\lambda_{1}\right)^{2}$ as shown in Figure 14, except at vertices with valency $N=3$.

\section{Numerical examples}

We implemented and tested our algorithm for computing pseudo-spline surfaces for a variety of input meshes. The shaded versions in Figures 1 and 9, as well as the reflection
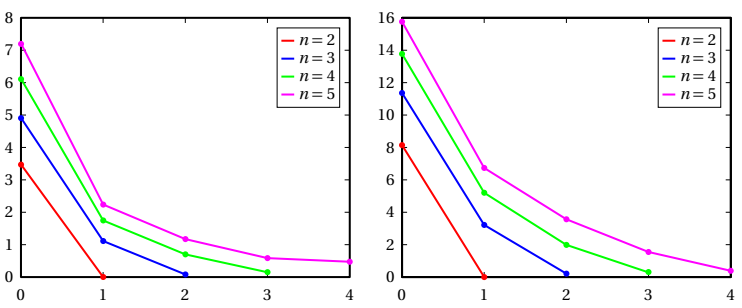

Figure 15: Average distance over l in per cent of the bounding box diagonal between control vertices and corresponding points on the limit surface for the meshes in Figure 1 (left) and Figure 16 (right).

lines and Gaussian curvature plots in Figure 13 show the behaviour of the limit surface for a fixed number of $n=4$ smoothing steps and varying $l$. On the one hand, $l=0$ gives a degree-7 B-spline surface, which is $C^{6}$ except at extraordinary vertices, but quite far from the control mesh. On the other hand, $l=3$ gives a surface analogue of the interpolatory 8-point scheme, which is $C^{3}$ around regular vertices and interpolates most of the control vertices. It is comparable to the surface generated by the 8-point scheme of Deng and Ma [DM13], which has the same smoothness, but our surface tends to give sharper edges and a smoother shape around regular vertices.

We observe that the smoothness as well as the distance between the control vertices and the limit surface vary intuitively between $l=0$ and $l=n-1$ (see Figure 15), thus providing a useful control parameter to the user. The other 

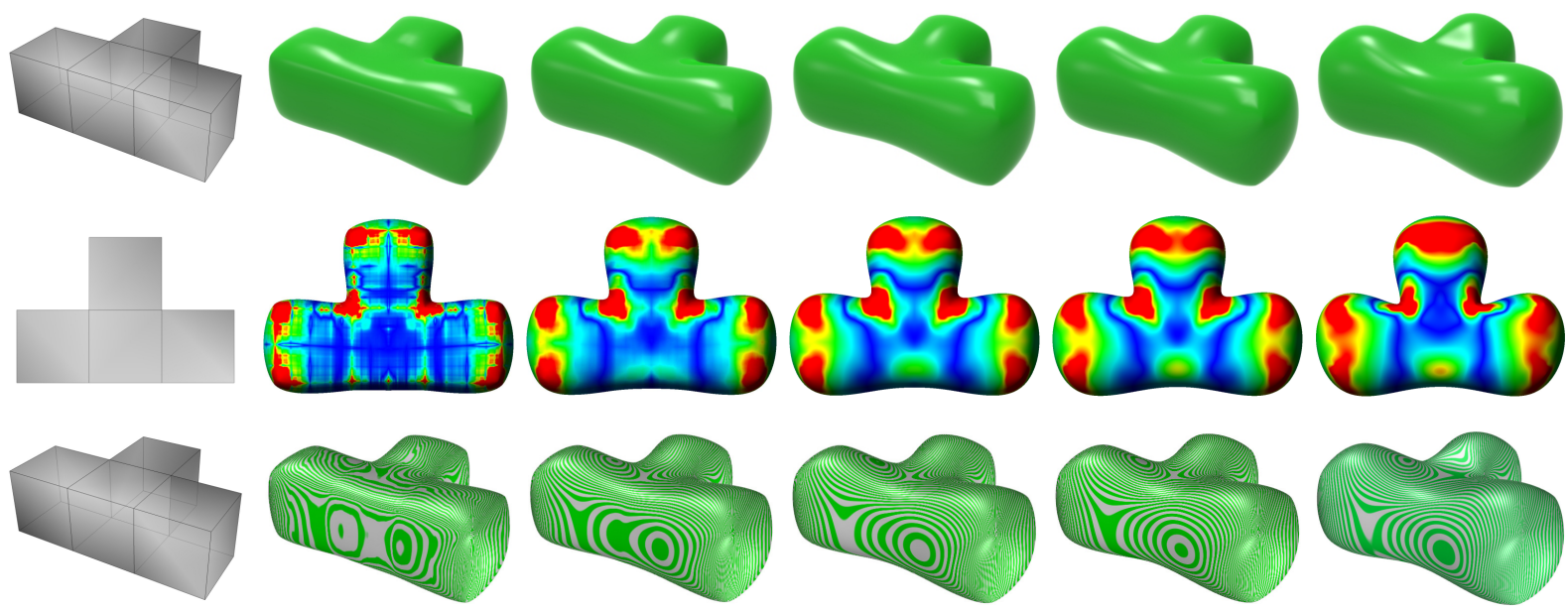

control mesh

$(n, l)=(2,1), C^{1}$

$(n, l)=(3,2), C^{2}$

$(n, l)=(4,3), C^{3}$

$(n, l)=(5,4), C^{4}$

$(n, l)=(6,5), C^{4}$

Figure 16: Interpolatory pseudo-spline surfaces with varying $n$ and $l=n-1$ for a T-shaped control mesh.
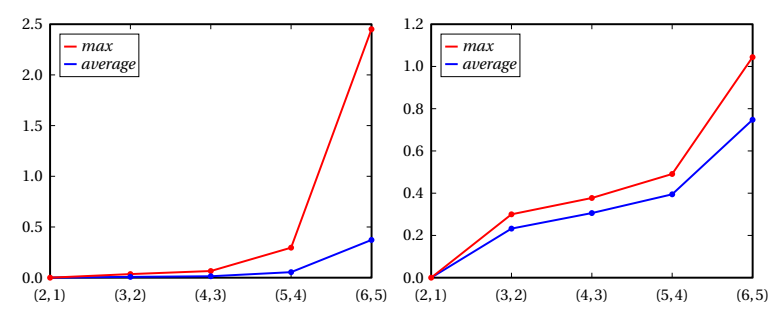

Figure 17: Maximal and average distance in per cent of the bounding box diagonal for different values of $(n, l)$ between extraordinary control vertices and corresponding points on the limit surface for the meshes in Figure 1 (left) and Figure 16 (right). The distance at regular vertices is zero.

control parameter $n$ can be used to generally increase the smoothness of the limit surfaces, as shown by Dong and Shen [DS07, Table 1] and Floater and Muntingh [FM13, Theorem 3], except at extraordinary vertices. For example, if the user desires interpolating limit surfaces, then increasing $n$ while choosing $l=n-1$ gives successively smoother results, as shown in Figure 16. As mentioned above, the resulting limit surfaces do not interpolate extraordinary control, and the distance increases with $n$ (see Figure 17).

A convenient consequence of our decomposition approach to generating pseudo-spline surfaces is that we can handle arbitrary polygonal input meshes, because the first local operation of the first subdivision step is midpoint refinement, which splits any initial polygonal face into quadrilaterals by connecting its barycentre with the edge midpoints. All subsequent local operations and subdivision steps then operate on quadrilaterals without the need to change anything in the algorithm. An example is shown in Figure 18.
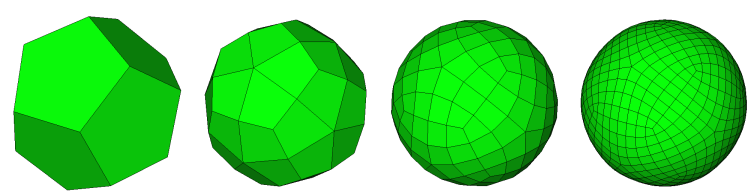

Figure 18: Three steps of the pseudo-spline subdivision scheme with $(n, l)=(6,5)$, applied to a dodecahedron.

\section{Conclusion}

We have shown in this paper how to generate pseudo-spline surfaces by iteratively subdividing an initial quadrilateral mesh with arbitrary topology. These subdivision surfaces are interesting for modelling, because they come with intuitive control parameters for blending between interpolating and approximating surfaces with arbitrarily high order of continuity in regular mesh regions and $C^{1}$-continuity at extraordinary vertices. This considerably enlarges the design space of possible limit surfaces and it is even possible to set these control parameters to different values in different parts of the initial mesh, as shown in Figure 19. It remains future work, however, to design appropriate subdivision rules for the transition regions to avoid the curvature artefacts visible in this example.

The advantage of our approach is that each subdivision step is based on the repeated application of the masks $\boldsymbol{\sigma}, \boldsymbol{\delta}$, and $\boldsymbol{\chi}$, which require only the information from the direct neighbourhood of each vertex, even though the overall subdivision masks can have large support and would be cumbersome to generalize to extraordinary vertices.

We believe that the locality of the masks is also advantageous for implementing the subdivision process on the GPU 


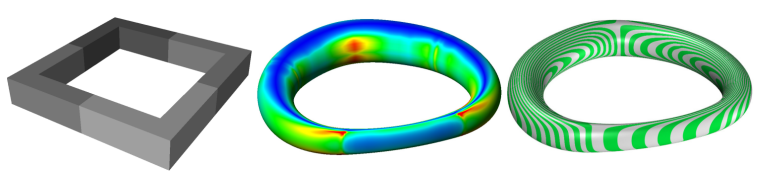

Figure 19: Pseudo-spline surface with varying parameters $n$ and $l$ in different parts: $(n, l)=(3,0)$ in the light grey part, $(n, l)=(3,1)$ in the adjacent grey part, and $(n, l)=(3,2)$ in the dark grey part.

and will investigate this matter in future work. We further plan to extend the method to handle boundaries and features like corners and creases.

A potential limitation of our approach is that the surface analogues of the interpolatory $2 n$-point schemes do not necessarily interpolate extraordinary input vertices, but it might be possible to fix this by adapting the weights $\gamma_{k}$ appropriately. It might further be possible to adapt the ideas of Augsdörfer et al. [ADS06] and to tune the weights $\gamma_{k}$ and the generalized local masks in Figure 7 such that the curvature of the limit surfaces becomes bounded.

\section{Acknowledgements}

This work is supported by NSFC (61370166, 61379072, 61003194), the Open Project Program of the State Key Lab of CAD\&CG (Grant No. A1304), Zhejiang University. We would like to thank Bay Raitt for the models used in Figure 1 and Figure 9 (top row), and Wenbiao Jin for helping with the visualization of the surfaces.

\section{References}

[ACDS09] Augsdörfer U. H., Cashman T. J., Dodgson N. A., SABIN M. A.: Numerical checking of $C^{1}$ for arbitrary degree quadrilateral subdivision schemes. In Mathematics of Surfaces XIII, vol. 5654 of LNCS. Springer, 2009, pp. 45-54. 8

[ADS06] AugSdöRfer U. H., Dodgson N. A., SAbin M. A.: Tuning subdivision by minimising Gaussian curvature variation near extraordinary vertices. Comput. Graph. Forum 25, 3 (2006), 263-272. 10

[CADS09] Cashman T. J., Augsdörfer U. H., Dodgson N. A., SABIN M. A.: NURBS with extraordinary points: highdegree, non-uniform, rational subdivision schemes. ACM Trans. Graph. 28, 3 (2009), Article 46, 9 pages. 2

[Cas12] Cashman T. J.: Beyond Catmull-Clark? A survey of advances in subdivision surface methods. Comput. Graph. Forum 31,1 (2012), 42-61.

[CC78] Catmull E., Clark J.: Recursively generated B-spline surfaces on arbitrary topology meshes. Comput.-Aided Des. 10, 6 (1978), 350-355. 7

[Dau92] DaUbechies I.: Ten Lectures on Wavelets. SIAM, 1992. 2

[DD89] Deslauriers G., Dubuc S.: Symmetric iterative interpolation processes. Constr. Approx. 5, 1 (1989), 49-68. 2
[DGL87] DYN N., GREgORY J. A., LEVIN D.: A four-point interpolatory subdivision scheme for curve design. Comput. Aided Geom. Des. 4, 4 (1987), 257-268. 2

[DHSS08] Dyn N., Hormann K., Sabin M. A., Shen Z.: Polynomial reproduction by symmetric subdivision schemes. $J$. Approx. Theory 155, 1 (2008), 28-42. 2

[DM13] DENG C., MA W.: A unified interpolatory subdivision scheme for quadrilateral meshes. ACM Trans. Graph. 32, 3 (2013), Article 23, 11 pages. 2, 7, 8

[DS07] Dong B., Shen Z.: Pseudo-splines, wavelets and framelets. Appl. Comput. Harmon. Anal. 22, 1 (2007), 78-104. 2,9

[Eir92] EIROLA T.: Sobolev characterization of solutions of dilation equations. SIAM J. Math. Anal. 23, 4 (1992), 1015-1030. 2

[FM13] FloAter M. S., Muntingh G.: Exact regularity of pseudo-splines. Tech. Rep. 1209.2692v2, arXiv, 2013. 2, 9

[Kob00] Kobbelt L.: $\sqrt{3}$-subdivision. In Proceedings of SIGGRAPH (2000), pp. 103-112. 2

[LLYL08] Lin S., Luo X., You F., Li Z.: Deducing interpolating subdivision schemes from approximating subdivision schemes. ACM Trans. Graph. 27, 5 (2008), Article 146, 7 pages. 2

[LM07] Li G., MA W.: A method for constructing interpolatory subdivision schemes and blending subdivision. Comput. Graph. Forum 26, 2 (2007), 185-201. 2, 7

[LR80] LANE J. M., RIESENFELD R. F.: A theoretical development for the computer generation and display of piecewice polynomial surfaces. IEEE Trans. Pattern Anal. Mach. Intell. 2, 1 (1980), 35-46. 1, 2

[MS01] Maillot J., Stam J.: A unified subdivision scheme for polygonal modeling. Comput. Graph. Forum 20, 3 (2001), 471479. 2

[OS03] Oswald P., SchrödER P.: Composite primal/dual $\sqrt{3}-$ subdivision schemes. Comput. Aided Geom. Des. 20, 3 (2003), 135-164. 2

[PC11] Prautzsch H., Chen Q.: Analyzing midpoint subdivision. Comput. Aided Geom. Des. 28, 7 (2011), 407-419. 2

[PR04] PETERS J., REIF U.: Shape characterization of subdivision surfaces-basic principles. Comput. Aided Geom. Des. 21, 6 (2004), 585-599. 8

[PR08] Peters J., ReIF U.: Subdivision Surfaces. Springer, 2008. 7

[Pra98] PRAUTZSCH H.: Smoothness of subdivision surfaces at extraordinary points. Adv. Comput. Math. 9, 3-4 (1998), 377389. 1

[RS08] Rossignac J., Schaefer S.: J-splines. Comput.-Aided Des. 40, 10-11 (2008), 1024-1032. 2, 3

[Sta01] STAM J.: On subdivision schemes generalizing uniform B-spline surfaces of arbitrary degree. Comput. Aided Geom. Des. 18, 5 (2001), 383-396. 1, 2

[SW03] SchaEFER S., WARREN J.: A factored interpolatory subdivision scheme for quadrilateral surfaces. In Curve and Surface Design: Saint-Malo 2002, Lyche T., Mazure M.-L., Schumaker L. L., (Eds.). Nashboro Press, 2003, pp. 373-382. 2

[WW01] WARREN J., WEIMER H.: Subdivision Methods for Geometric Design: A Constructive Approach. Morgan Kaufmann, 2001. 1, 3

[ZS01] ZORIN D., SCHRÖDER P.: A unified framework for primal/dual quadrilateral subdivision schemes. Comput. Aided Geom. Des. 18, 5 (2001), 429-454. 1, 4, 7 